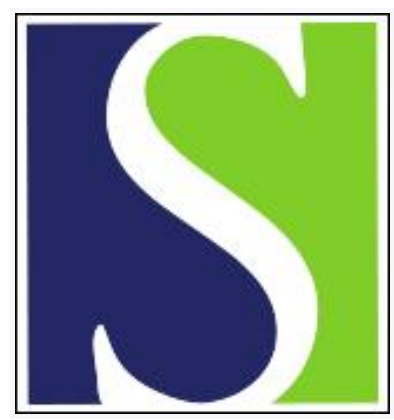

Scand J Work Environ Health 2003;29(3):171-188

https://doi.org/10.5271/sjweh.720

Issue date: Jun 2003

\title{
Long workhours and health
}

by van der Hulst $M$

Affiliation: Department of Work and Organizational Psychology, University of Nijmegen, PO Box 9104, 6500 HE Nijmegen, The Netherlands. secr.a\&o@psych.kun.nl

Refers to the following texts of the Journal: 1996;22(5):353-359 1997;23(6):403-413 1996;22(2):124-132 2000;26(4):306-316

The following articles refer to this text: $2003 ; 29(3): 167-169$;

2003;29(5):325-327; 2004;30(2):81-83; 2006;32(5):349-358; SJWEH Supplements 2006;(2):22-26; 2006;32(6):413-419; 2006;32(6):421-430; 2006;32(6):482-492; 2006;32(6):502-514; 2007;33(1):1-3; 2007;33(1):37-44; 2007;33(2):105-113; 2007;33(5):344-350; 2008;34(3):213-223; SJWEH Supplements 2008;(5):14-21; 2011;37(5):418-426; 2012;38(1):56-64; 2013;39(4):369-378; 2013;39(5):515-520; 2016;42(2):135-143; 2017;43(6):569-577; 2017;43(6):578-586; 2018;44(3):239-250; 2023;49(1):75-83

Key terms: cardiovascular disease; diabetes; empirical study; fatigue; health; health-related behavior; immunology; life-style; long workhours; overtime; physiology; recovery; review; systematic review

This article in PubMed: www.ncbi.nlm.nih.gov/pubmed/12828387

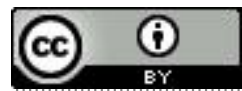




\title{
Long workhours and health
}

\author{
by Monique van der Hulst, $P h D^{1}$
}

van der Hulst M. Long workhours and health. Scand J Work Environ Health 2003;29(3):171-188.

\begin{abstract}
This paper summarizes the associations between long workhours and health, with special attention for the physiological recovery and behavioral life-style mechanisms that may explain the relationship. The evidence for these mechanisms has not been systematically reviewed earlier. A total of 27 recent empirical studies met the selection criteria. They showed that long workhours are associated with adverse health as measured by several indicators (cardiovascular disease, diabetes, disability retirement, subjectively reported physical health, subjective fatigue). Furthermore, some evidence exists for an association between long workhours and physiological changes (cardiovascular and immunologic parameters) and changes in health-related behavior (reduced sleep hours). Support for the physiological recovery mechanism seems stronger than support for the behavioral lifestyle mechanism. However, the evidence is inconclusive because many studies did not control for potential confounders. Due to the gaps in the current evidence and the methodological shortcomings of the studies in the review, further research is needed.
\end{abstract}

Key terms cardiovascular disease, diabetes, empirical studies, fatigue, health-related behavior, immunology, life-style, overtime, physiology, recovery, systematic review.

In reaction to a political conflict in the European Union concerning legislation on long workhours around 1995, several articles were published that addressed the relationship between extended workhours on the one hand and health and safety issues on the other (1-4). The main conclusion of these papers was that there were indeed good reasons to be concerned about the possible detrimental effects of extended workhours on health and safety, but that the evidence was inconclusive. Although the relationship between workhours and health has been studied extensively (5-9), the majority of the studies addressing this subject focused on the effects of shift work rather than on the effects of long workhours. The literature on extended shifts and compressed workweeks is certainly relevant for knowledge about the effects of long workhours on health. However, it can be difficult to disentangle the effects of the scheduling of the shiftunusual hours of work that may cause sleep loss and a disturbance of circadian rhythms (10) — and the length of the shift. In extreme cases, extensive overtime may be associated with sleep loss and disturbance of body rhythms [eg, possibly in the case of junior doctors and karoshi victims (11-13)], but it is not likely to occur if one works a few extra hours every day. Thus, halfway through the 1990s, studies addressing the effects of long workhours independently of shift work were relatively scarce.

As has already been stated, the existing literature suggests that long workhours are associated with adverse health, but the evidence is not very strong. Sparks and her colleagues (3) found a mean correlation of 0.13 between weekly workhours and overall ill health in a meta-analysis based on 21 studies. A further categorization of the health indicators resulted in a mean correlation of 0.06 with physiological ill health (although within this category the correlations with cardiovascular complaints were much higher, 0.20 and 0.51 ) and a mean correlation of 0.15 with psychological ill health. Thus long workhours seem to be associated with poor health, but the association is rather weak, although there may be stronger relationships for specific health indicators. A particularly important issue with respect to the strength of the evidence is possible confounding between long workhours and other factors that might

1 Department of Work and Organizational Psychology, University of Nijmegen, The Netherlands.

Reprint requests to: Dr Monique van der Hulst, Department of Work and Organizational Psychology, University of Nijmegen, PO Box 9104, 6500 HE Nijmegen, The Netherlands. [E-mail: secr.a\&o@psych.kun.nl] 
affect health, such as demographic variables, work characteristics, home characteristics, and personality. As was pointed out by Spurgeon and her co-workers (4), long workhours are likely to coincide with high job demands. A difference in workload between employees who work extended hours and those who do not might explain possible differences in health. Other psychosocial work characteristics, such as job control and social support (14-16), may also covary with workhours. Furthermore, in some studies, the number of hours worked is used as an indicator of task demands. This approach makes it impossible to disentangle the effects of job demands and long workhours. Thus simple correlations between workhours and health can only provide weak evidence. Perhaps the most important reason why the evidence for a relationship between long workhours and adverse health is inconclusive is that many studies have not controlled for covariates.

Extended workhours may affect health through different pathways. First, long hours of work imply that one has to invest effort in the worktask for a longer period of time, whereas the time available for recovery is reduced $(17,18)$. Insufficient recovery is thought to disturb physiological processes (blood pressure, hormone excretion, sympathetic nervous system activity) and, eventually, lead to psychological and physical health complaints $(19,20)$. Second, long workhours are thought to be associated with life-style factors such as smoking and coffee and alcohol consumption, unhealthy diet, and lack of exercise (21-23). In turn, unhealthy behavior causes physiological changes (eg, high blood pressure, high cholesterol) and increased risk for cardiovascular disease and adverse health in general. The physiological recovery mechanism and the behavioral life-style mechanism are also thought to be involved in the relationship between shift work and health, especially cardiovascular risk (24). It should be pointed out that these processes are not mutually exclusive and may operate simultaneously. Moreover, the mechanisms involved may differ for different types of complaints. Although several authors [eg, Sparks et al (3) and Steenland (25)] have speculated about the mechanisms that may explain associations between long workhours and adverse health, the evidence for changes in physiological processes and health-related behavior is rather scarce.

My review provides an update of current knowledge based on recent studies concerning long workhours and health by considering new evidence for the association between long workhours and a range of health indicators and systematically investigating the methodological characteristics of these studies. The purpose of the review was to provide an up-to-date overview of recent empirical research on extended workhours and health and also an overview of the main conclusions of these studies, taking the methodological quality of the studies into account. As has already been stated, only evidence from studies that controlled for possible covariates can provide strong evidence for associations between long workhours and health. Moreover, this review examined the existence of two specific pathways (the physiological recovery mechanism and the behavioral life-style mechanism) that might explain the relationship between extended workhours and adverse health. The evidence for these mechanisms has not been systematically reviewed earlier. Because of the diversity in the measurement of extended hours, the types of outcome variables used, and the variety in study designs, it was decided to conduct a systematic narrative review rather than a meta-analysis. The following three research questions guided the review: (i) is there evidence in the recent literature for a relationship between long workhours and specific aspects of health and how strong is this evidence, (ii) is there evidence in the recent literature for physiological changes in persons who work long hours, and (iii) does the recent literature contain evidence of changes in health-related behavior in persons who work long hours?

\section{Subjects and methods}

\section{Search strategy}

The review was limited to articles published in peer-reviewed psychological and medical journals in the English language. On-line searches were carried out in the PsycInfo and Medline databases, from January 1996 to June 2001. As a means of minimizing overlap with earlier reviews (3, 4), 1996 was chosen as the year to start the search. The following key words were used: work(ing) hours, long hours, extended hours, overtime, and overwork. The author examined the abstracts of all references in order to decide whether the study was relevant according to the selection criteria. All potentially relevant articles were retrieved and read.

\section{Inclusion criteria}

Type of study. Only empirical studies were included in the review, with the exception of case studies.

Sample. The study population had to be a working population or a patient group with a documented work history. At least a part of the study population should have worked more than 40 hours a week. Studies in which the mean number of workhours a week plus one standard deviation was lower than 40 were excluded, as well as studies that only compared part-time and full-time employees. Studies that explicitly focused on the effects 
of compressed workweeks were excluded as well. Studies with populations working irregular hours or shift work were only included if at least part of the population worked normal office hours and if shift work and overtime were included in the analyses separately.

Measurement of extended hours. Only studies that reported workhours (per day or per week) or overtime hours (per day, week, month or year) were included. Studies that measured the frequency of overtime work or that only reported whether or not participants worked overtime were excluded. Extended hours had to be measured on the individual level; studies that focused on organizational-level assessment of the prevalence of overtime or estimated the prevalence of overtime based on the participants' occupation were excluded.

Outcome measures. In order to keep the review focused, only studies that concerned health status (mortality, diagnosed disease, work disability, subjective health), physiological indices, or health-related behavior were included. In the psychological literature, several studies concerning effects of extended workhours on job satisfaction, job involvement, work-family conflict, well-being of partners and children, performance, and accidents were retrieved, but these were not considered relevant for this study. In the medical literature, several studies that focused on reproductive health were considered irrelevant for the review and were therefore excluded.

Statistical analysis. In order to be included, studies needed to explicitly test the association between extended hours and the outcome measure. The strength of the relationship and the statistical technique used to test this relationship had to be reported clearly. Studies focusing on the antecedents of extended workhours and purely descriptive studies were excluded. Furthermore, there were many studies that addressed questions such as "What do workers find stressful about their jobs?" or "Do long hours affect your health?" These studies focus on the factors to which workers attribute their health complaints instead of on statistical associations and were therefore excluded. Finally, comparisons of health indicators for groups that happened to differ in mean workhours but were categorized on the the basis of some other criterion (eg, job type) were not included in the review, because such studies can only produce circumstantial evidence.

A total of 27 studies met the selection criteria (2652) (table 1). In one case, two articles $(49,50)$ were written based on the same sample but with different dependent measures. Two other articles $(39,40)$ concerned follow-up studies on the same population, one after 3 years and one after 5 years. It should be kept in mind that the results of these studies are not statistically independent. Furthermore, one paper (30) described a between-group comparison and a within-group comparison in different samples. The two comparisons are considered independent studies and are summarized separately in the table. In summary, the final selection included 27 articles reporting about 28 studies in 26 independent samples. Table 1 describes the methodological characteristics and the results of the studies. The next section lists the characteristics of the studies that were summarized in the table.

\section{Methodological characteristics and results}

Sample. The following characteristics of the sample are mentioned in the table: number of participants, country of residence, percentage of men and women, mean age of the sample (or age range if mean age was not reported), type of occupation(s), and type of organization(s). The selected studies concerned 9 European samples (26-28, 36, 41, 43, 47, 49, 50, 51), 4 samples from the United States $(29,32,42,48)$, and 13 Japanese samples (30, 31, 33-35, 37-40, 44-46, 52). Twelve studies had mixed-gender samples $(26,27,29,32,34,41-43,47-$ 51 ), although one was predominantly male (32) and one predominantly female (48). The other 14 studies had allmale samples $(28,30,31,33,35-40,44-46,52)$.

Time dimension. The test of the association between extended workhours and the outcome measures could be cross-sectional, case-referent, longitudinal, or repeated measures. In some studies, the sample came from a study with a longitudinal design, but the relationship between extended hours and outcome measures was only studied on the basis of data collected at a single point in time (although other relationships were studied longitudinally). This was particularly the case when long workhours were not the primary focus of the study. All the longitudinal studies tested whether long workhours at baseline or the average amount of workhours during the period of study were associated with changes in health status during the follow-up period. Thus these studies did not investigate whether within-subject changes in workhours were associated with within-subject changes in health. Repeated measures designs were defined as natural experiments that made within-subject comparisons between outcome variables in relatively long workhour periods and outcome variables in relatively short workhour periods. Most of the studies were cross-sectional (26, 27, 29, 30, $31-34,37,42-45,48-52)$. Two studies had a case-referent design $(28,46)$. There were five independent longitudinal studies $(35,36,38-41)$, and two repeatedmeasures studies $(30,47)$. 
Table 1. Overview of the methodological characteristics and results of the 27 selected studies $\left[A_{m}=\right.$ mean age, $A_{m b}=$ mean age at baseline in longitudinal study, $A=$ age range, $A_{b}=$ age range at baseline in longitudinal study, $A N C O V A=$ analysis of covariance, $A N O V A$ = analysis of variance, $\mathrm{BMI}=$ body mass index, Cat = categories, Cor =correlation, Con = controlled for the following (categories of) covariates or confounders, DBP= diastolic blood pressure, Dich=dichotomous, Fish=Fisher's exact test, GHQ=General Health Questionnaire, $\mathrm{HF}=$ high frequency, $\mathrm{HR}=$ heart rate, $\mathrm{HRV}=$ heart rate variability, hyper = hypertensive group, $\mathrm{Ig}=\mathrm{immunoglobulin}, \mathrm{IHD}=$ ischemic heart disease, Int = interaction effects tested for particular moderator variables, LWH=long workhours, MLinRA=multivariate linear regression analysis, MLogRA=multivariate logistic regression analysis, MRegA=multi-level regression analysis, multi = multivariate association (after correction for covariates), $\mathrm{N}_{\text {case }}=$ number of cases, normotens = normotensive group, $\mathrm{NR}=$ not reported, $\mathrm{N}_{\text {ref }}=$ number of referents, $\mathrm{Obj}=$ objective, registered workhours, Occup = type of occupation, $\mathrm{OF}=$ overtime frequency, $\mathrm{OH}=$ overtime hours, $\mathrm{Organ}=$ type of organization, $\mathrm{PC}=$ personal computer, range=range of overtime hours, either range or mean and standard deviation (SD), Rcor $=$ rank correlation, RMANOVA= repeated measurements analysis of variance, $\mathrm{SA}=$ separate analyses for particular groups (ie, relationship between extended hours and outcome variables studied separately for particular groups, usually gender or age), SBP=systolic blood pressure, SRWH=self-reported workhours, SWH=short workhours, ULogRA=univariatelogistic regression analysis, uni =univariate association (only reported if the association was not tested by means of a multivariate analysis technique), UK=United Kingdom, US= United States, VDT = video display terminal, $\mathrm{WH}=$ =workhours, Wil =Wilcoxon rank-sum test, ${ }^{\text {ws }}=$ within-subject association, $+=$ significant positive relationship, $-=$ significant negative relationship, $0=$ no significant relationship]

\begin{tabular}{|c|c|c|c|c|c|c|}
\hline Study & Sample & Time dimension & Statistical analysis & $\begin{array}{l}\text { Measurement of } \\
\text { extended hours }\end{array}$ & Outcome measures & Results \\
\hline \multirow[t]{2}{*}{$\begin{array}{l}\text { Baldwin et al, } \\
1997(26)\end{array}$} & \multirow[t]{2}{*}{$\begin{array}{l}\mathrm{N}=142, \mathrm{UK} \\
\text { males: } 55 \% \text {, } \\
\mathrm{A}_{\mathrm{m}}: 25 \text { years, } \\
\text { Occup: doctors } \\
\text { (senior house } \\
\text { officers), Organ: } \\
\text { hospitals }\end{array}$} & \multirow[t]{2}{*}{$\begin{array}{l}\text { Oross-sectional: } \\
\text { test of linear relation }\end{array}$} & \multirow[t]{2}{*}{$\begin{array}{l}\text { Cor \& MLinRA, Con: } \\
\text { significant associations } \\
\text { controlled for each } \\
\text { other }\end{array}$} & \multirow[t]{2}{*}{$\begin{array}{l}\text { SRWH, WH/week } \\
\text { (preceding week, } \\
\text { including on call), } \\
\text { mean } 76 \text {, range } \\
\text { 33-128 }\end{array}$} & $\begin{array}{l}\text { Subjective health } \\
\text { Total GHQ } \\
\text { Somatic symptoms } \\
\text { Severe depression } \\
\text { Psychosomatic symptoms } \\
\text { Social dysfunction } \\
\text { Physical ailments last year }\end{array}$ & $\begin{array}{l}\text { Ouni } \\
+^{\text {multi }} \\
0^{\text {uni }} \\
\text { Ouni }^{\text {uni }} \\
+^{\text {multi }} \\
\text { Ouni }^{\text {uni }}\end{array}$ \\
\hline & & & & & $\begin{array}{l}\text { Behavior } \\
\text { Alcohol consumption (last } 7 \\
\text { days) }\end{array}$ & _uni \\
\hline $\begin{array}{l}\text { Borg \& } \\
\text { Kristensen, } \\
1999(27)\end{array}$ & $\begin{array}{l}\mathrm{N}=1306, \\
\text { Denmark, } \\
\text { males: } 90 \% \text {, } \\
\mathrm{A}_{\mathrm{m}}: 41.5 \text { years, } \\
\text { Occup: traveling } \\
\text { salespeople, } \\
\text { Organ: diverse }\end{array}$ & $\begin{array}{l}\text { Oross-sectional: } \\
\text { test of linear relation }\end{array}$ & $\begin{array}{l}\text { MLinRA, Con: demo- } \\
\text { graphic variables and } \\
\text { psychosocial job } \\
\text { characteristics }\end{array}$ & $\begin{array}{l}\text { SRWH, WH/week, } \\
\text { mean } 43.9, \text { SD } 8.5\end{array}$ & $\begin{array}{l}\text { Subjective health } \\
\text { Mental health }\end{array}$ & $0^{\text {multi }}$ \\
\hline $\begin{array}{l}\text { Emdad et al, } \\
1998(28)\end{array}$ & $\begin{array}{l}\mathrm{N}_{\text {case }}=13, \\
\mathrm{~N}_{\text {ref }}=56, \\
\text { Sweden, } \\
\text { males: } 100 \% \text {, } \\
\text { A: } 25-52 \text { years, } \\
\text { Occup: profes- } \\
\text { sional drivers, } \\
\text { Organ: diverse }\end{array}$ & $\begin{array}{l}\text { Case-referent: } \\
\text { cases: IHD; refer- } \\
\text { ents: hypertensive, } \\
\text { borderline hyperten- } \\
\text { sive, normotensive; } \\
\text { all with same occupa- } \\
\text { tion }\end{array}$ & $\begin{array}{l}\text { ANOVA \& MLinRA, } \\
\text { Con: other risk factors }\end{array}$ & $\begin{array}{l}\text { SRWH, WH/day, } \\
\text { IHD cases, } \\
\text { mean } 8.5, \text { SD } 2.6\end{array}$ & $\begin{array}{l}\text { Disease } \\
\text { IHD (hospital treatment } \\
\text { in coronary care unit } \\
\text { within last } 2 \text { years) } \\
\text { Behavior } \\
\text { BMl } \\
\text { Smoking }\end{array}$ & $\begin{array}{l}+^{\text {uni }} \\
+^{\text {multi }} \\
0^{\text {multi }}\end{array}$ \\
\hline $\begin{array}{l}\text { Etner \& } \\
\text { Grzywacz, } \\
2001(29)\end{array}$ & $\begin{array}{l}\mathrm{N}=2048, \mathrm{US}, \\
\text { males: } 50 \% \text {, } \\
\text { A: } 25-74 \text { years, } \\
\text { Occup: diverse, } \\
\text { Organ: diverse }\end{array}$ & $\begin{array}{l}\text { Cross-sectional: } \\
\text { comparison of three } \\
\text { groups varying in } \\
\text { workhours }\end{array}$ & $\begin{array}{l}\text { MLogRA, Con: occu- } \\
\text { pation-based and } \\
\text { self-reported job } \\
\text { characteristics }\end{array}$ & $\begin{array}{l}\text { SRWH, WH/week, } \\
\text { Cat: }<35,35-45 \text {, } \\
>45\end{array}$ & $\begin{array}{l}\text { Subjective health } \\
\text { Unambiguously negative } \\
\text { impact of work on health }\end{array}$ & $t^{\text {multi }}$ \\
\hline $\begin{array}{l}\text { Hayashi et al, } \\
1996(30)\end{array}$ & $\begin{array}{l}\mathrm{N}=47 \text {, Japan, } \\
\text { males: } 100 \% \text {, } \\
\mathrm{A}_{\mathrm{m}}: 39-47 \\
\text { years, for } \\
\text { different groups, } \\
\text { Occup: white } \\
\text { collar workers, } \\
\text { Organ: electrical } \\
\text { goods manufac- } \\
\text { turer }\end{array}$ & $\begin{array}{l}\text { Cross-sectional: } \\
\text { comparison of two } \\
\text { groups varying in } \\
\text { overtime hours }\end{array}$ & $\begin{array}{l}\text { t-tests: Wil \& Fish, SA: } \\
\text { normotensives and } \\
\text { mild hypertensives }\end{array}$ & $\begin{array}{l}\text { SRWH?, OH/month } \\
\text { Dich: } \leq 30, \geq 60\end{array}$ & $\begin{array}{l}\text {, Subjective health } \\
\text { Fatigue symptoms: } \\
\text { before work } \\
\text { after work } \\
\text { Physiology } \\
\text { SBP (occasional) } \\
\text { DBP (occasional) } \\
\text { Blood samples: } \\
\text { cholesterol (2 indices) } \\
\text { blood sugar } \\
\text { fructosamine } \\
\text { Urea nitrogen } \\
\text { Creatinine } \\
\text { SBP 24-hour } \\
\text { DBP 24-hour } \\
\text { HR 24-hour }\end{array}$ & $\begin{array}{l}+^{\text {uni }} \\
\left.+^{\text {uni }} \text { (mild hyper }\right) \\
\text { Ouni }^{\text {uni }} \\
\text { Ouni }^{\text {uni }} \\
\text { Ouni }^{\text {uni (normotens) }} \\
+^{\text {uni }} \\
\text { Ouni }^{\text {uni }} \\
\text { Ouni }^{\text {uni (normotens) }}{ }^{\text {a }} \\
\text { Ouni a }^{\text {uni }} \\
\text { Ouni a }^{\text {un }}\end{array}$ \\
\hline & & & & & $\begin{array}{l}\text { Behavior } \\
\text { BMl } \\
\text { Sleeping hours } \\
\text { Smoking } \\
\text { Alcohol consumption }\end{array}$ & $\begin{array}{l}\text { Ouni } \\
\text { _uni (mild hyper) } \\
\text { Ouni } \\
\text { Ouni }\end{array}$ \\
\hline
\end{tabular}


Table 1. Continued

\begin{tabular}{|c|c|c|c|c|c|c|}
\hline Study & Sample & Time dimension & Statistical analysis & $\begin{array}{l}\text { Measurement of } \\
\text { extended hours }\end{array}$ & Outcome measures & Results \\
\hline $\begin{array}{l}\text { Hayashi et al, } \\
1996(30)\end{array}$ & $\begin{array}{l}\mathrm{N}=19 \text {, Japan, } \\
\text { males: } 100 \% \text {, } \\
\text { A }_{\mathrm{m}}: 36 \text { years, } \\
\text { Occup: white- } \\
\text { collar workers } \\
\text { of accounting } \\
\text { department, } \\
\text { Organ: electrical } \\
\text { goods manu- } \\
\text { facturer }\end{array}$ & $\begin{array}{l}\text { Repeated measures: } \\
2 \text { points in time, time } \\
\text { span not reported, } \\
\text { comparison of busy } \\
\text { period and reference } \\
\text { period }\end{array}$ & t-tests, Wil & $\begin{array}{l}\text { SRWH?, OH/month, } \\
\text { mean: } 96 \text { for busy } \\
\text { period \& } 43 \text { for } \\
\text { reference period }\end{array}$ & $\begin{array}{l}\text {, Subjective health } \\
\text { Fatigue symptoms } \\
\text { Physiology } \\
\text { SBP } \\
\text { DBP } \\
\text { HR(24-hour registration) } \\
\text { Behavior } \\
\text { Sleep hours } \\
\text { Smoking }\end{array}$ & $\begin{array}{l}+^{\text {ws }} \\
++^{\text {ws a }} \\
+ \text { ws a } \\
+{ }^{\text {ws a }} \\
\text { - }^{\text {ws }}\end{array}$ \\
\hline $\begin{array}{l}\text { Iwasaki et al, } \\
1998 \text { (31) }\end{array}$ & $\begin{array}{l}\mathrm{N}=71 \text {, Japan, } \\
\text { males: } 100 \% \text {, } \\
\text { A: } 22-60 \text { years, } \\
\text { Occup: salesmen, } \\
\text { Organ: machinery } \\
\text { manufacturing } \\
\text { company }\end{array}$ & $\begin{array}{l}\text { Cross-sectional: } \\
\text { comparison of two } \\
\text { groups varying in } \\
\text { number of workhours }\end{array}$ & t-tests, SA: age & $\begin{array}{l}\text { SRWH, WH/week } \\
\text { (office + oneway } \\
\text { commuting time), } \\
\text { Dich (median split), } \\
\text { mean: } 57.2 \text { for } \\
\text { SWH group \& } 64.5 \\
\text { for LWH group }\end{array}$ & $\begin{array}{l}\text { Subjective health } \\
\text { Fatigue symptoms: } \\
\text { drowsiness or dullness } \\
\text { difficulty concentrating } \\
\text { Feeling physical abnormality } \\
\text { Physiology } \\
\text { HRV } \\
\text { SBP } \\
\text { Serum cholesterol }\end{array}$ & 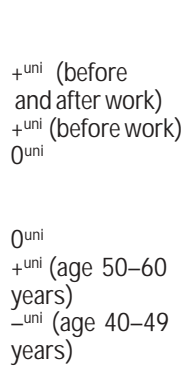 \\
\hline $\begin{array}{l}\text { Jex \& Bliese, } \\
1999 \text { (32) }\end{array}$ & $\begin{array}{l}\mathrm{N}=2273, \mathrm{US} \\
\text { males: } 96 \%, \\
\mathrm{~A}_{\mathrm{m}}: 25 \text { years, } \\
\text { Occup: soldiers, } \\
\text { Organ: } 36 \text { army } \\
\text { companies }\end{array}$ & $\begin{array}{l}\text { Cross-sectional: } \\
\text { test of linear relation }\end{array}$ & $\begin{array}{l}\text { MRegA, Int: self-efficacy } \\
\text { and collective efficacy, } \\
\text { Con: several job } \\
\text { stressors }\end{array}$ & $\begin{array}{l}\text { SRWH, WH/day } \\
\text { (past week), mean } \\
10.23, \text { SD } 2.85\end{array}$ & $\begin{array}{l}\text { Subjective health } \\
\text { Psychological strain } \\
\text { Physical strain }\end{array}$ & $\begin{array}{l}+^{\text {multi b }} \\
+^{\text {multi }}\end{array}$ \\
\hline $\begin{array}{l}\text { Kageyama } \\
\text { et al, } 1998 \\
(33)^{c}\end{array}$ & $\begin{array}{l}\mathrm{N}=223, \text { Japan, } \\
\text { males: } 100 \% \text {, } \\
\text { A }_{\mathrm{m}}: 30.8 \text { years, } \\
\text { Occup: white- } \\
\text { collar workers, } \\
\text { Organ: private } \\
\text { company }\end{array}$ & $\begin{array}{l}\text { Cross-sectional: } \\
\text { comparison of } \\
\text { three groups } \\
\text { varying in over- } \\
\text { time hours }\end{array}$ & $\begin{array}{l}\text { Rcor \& ANCOVA, Con: } \\
\text { age, BMl, current } \\
\text { smoking status, current } \\
\text { alcohol consumption }\end{array}$ & $\begin{array}{l}\text { SRWH, OH/month } \\
\text { Cat: <20, 20-59, } \\
\geq 60\end{array}$ & $\begin{array}{l}\text { Physiology } \\
\text { HRV: } \\
\text { vagal activity during supine rest } \\
\text { sympathetic activity during } \\
\text { supine rest } \\
\text { vagal + sympathetic activity } \\
\text { during supine rest } \\
\text { vagal activity during standing } \\
\text { rest } \\
\text { sympathetic activity during } \\
\text { standing rest } \\
\text { vagal + sympathetic activity } \\
\text { during standing rest } \\
\text { Behavior } \\
\text { Sleep hours on weekdays } \\
\text { Sleep hours before holidays } \\
\text { Frequency of exercise }\end{array}$ & $\begin{array}{l}0^{\text {multi }} \\
0^{\text {multi }} \\
0^{\text {multi }} \\
\text { _multi }^{\text {multi }} \\
0^{\text {multi }}\end{array}$ \\
\hline $\begin{array}{l}\text { Kageyama } \\
\text { et al, } 2001 \\
\text { (34) }\end{array}$ & $\begin{array}{l}\mathrm{N}=260 \text {, Japan, } \\
\text { males: } 89 \% \text {, } \\
\text { A: } 20-59 \text { years, } \\
\text { Occup: white } \\
\text { collar workers } \\
\text { (editors, sales } \\
\text { persons, clerks), } \\
\text { Organ: private } \\
\text { publishing } \\
\text { company }\end{array}$ & $\begin{array}{l}\text { Oross-sectional: } \\
\text { comparison of three } \\
\text { groups varying in } \\
\text { overtime hours }\end{array}$ & $\begin{array}{l}\text { MLinRA, Con: work- } \\
\text { load and commuting } \\
\text { time or workload and } \\
\text { age }\end{array}$ & $\begin{array}{l}\text { SRWH, OH/month } \\
\text { in past } 3 \text { months, } \\
\text { Cat: } 0-59,60-79 \text {, } \\
\geq 80\end{array}$ & $\begin{array}{l}\text { Behavior } \\
\text { Sleep length weekdays } \\
\text { Sleep debt weekdays }\end{array}$ & $t^{\text {multiti }}$ \\
\hline $\begin{array}{l}\text { Kawakami } \\
\text { et al, } 1999 \\
\text { (35) }\end{array}$ & $\begin{array}{l}\mathrm{N}=2194, \\
\text { Japan, males: } \\
100 \% \text {, } \mathrm{A}_{\mathrm{b}}: 18- \\
60 \text { years, Occup: } \\
\text { industrial work- } \\
\text { ers, Organ: elec- } \\
\text { trical company }\end{array}$ & $\begin{array}{l}\text { Longitudinal: } \\
\text { comparison of three } \\
\text { groups varying in } \\
\text { overtime hours } \\
\text { at baseline, } \\
\text { baseline } 1984, \\
\text { follow-up } 1992\end{array}$ & $\begin{array}{l}\text { MLogRA,Con: demo- } \\
\text { graphic, behavioral } \\
\text { life-style, family } \\
\text { history of diabetes }\end{array}$ & $\begin{array}{l}\text { SRWH, OH/month } \\
\text { in past month, at } \\
\text { baseline, Cat: 0-25, } \\
25-50,>50\end{array}$ & $\begin{array}{l}\text { Disease } \\
\text { Diagnosed noninsulin } \\
\text { dependent diabetes mellitus } \\
\text { according to WHO criteria } \\
\text { (urine sample) during } \\
\text { follow-up }\end{array}$ & $t^{\text {multi }}$ \\
\hline
\end{tabular}


Table 1. Continued

\begin{tabular}{|c|c|c|c|c|c|c|}
\hline Study & Sample & Time dimension & Statistical analysis & $\begin{array}{l}\text { Measurement of } \\
\text { extended hours }\end{array}$ & Outcome measures & Results \\
\hline $\begin{array}{l}\text { Krause et al, } \\
1997(36)\end{array}$ & $\begin{array}{l}\mathrm{N}=968, \\
\text { Finland, } \\
\text { males: } 100 \% \text {, } \\
\mathrm{A}_{\mathrm{mb}}: \text { cohorts \& } \\
42,48,54,60 \\
\text { years of age, } \\
\text { Occup: diverse, } \\
\text { Organ: diverse }\end{array}$ & $\begin{array}{l}\text { Longitudinal: } \\
\text { 4-year follow-up, } \\
\text { baseline between } \\
1984 \text { and } 1989, \\
\text { comparison of four } \\
\text { groups varying in } \\
\text { workhours }\end{array}$ & $\begin{array}{l}\text { MLogRA, } \\
\text { Con: age, economic } \\
\text { factors, prevalent } \\
\text { diseases, and } \\
\text { health behavior }\end{array}$ & $\begin{array}{l}\text { SRWH, WH/week } \\
\text { at baseline?, Cat: } \\
<40,40-44 \text {, } \\
\text { 45-59, } \geq 60\end{array}$ & $\begin{array}{l}\text { Work diability } \\
\text { Disability retirement }\end{array}$ & $t^{\text {multi }}$ \\
\hline $\begin{array}{l}\text { Mure et al, } \\
1996 \text { (37) }\end{array}$ & $\begin{array}{l}\mathrm{N}=69, \text { Japan, } \\
\text { males: } 100 \% \text {, } \\
\mathrm{A}_{\mathrm{m}}: 44.2 \text { years, } \\
\text { Occup: diverse, } \\
\text { Organ: metal plant }\end{array}$ & $\begin{array}{l}\text { Cross-sectional: } \\
\text { comparison of two } \\
\text { groups varying in } \\
\text { workhours }\end{array}$ & $\begin{array}{l}\text { MLogRA, Con: life- } \\
\text { style factors }\end{array}$ & $\begin{array}{l}\text { SRWH, WH/day, } \\
\text { Dich: }<9, \geq 9\end{array}$ & $\begin{array}{l}\text { Physiology } \\
\text { Urinary mutagens or } \\
\text { carcinogens in 24-hour } \\
\text { specimens ( } 9 \text { fractions) }\end{array}$ & $0^{\text {multi }}$ \\
\hline $\begin{array}{l}\text { Nakamura } \\
\text { et al, } 1998 \\
\text { (38) }\end{array}$ & $\begin{array}{l}\mathrm{N}=248 \text {, Japan, } \\
\text { males: } 100 \% \text {, } \\
\mathrm{A}_{\mathrm{m}}: 30.9 \text { years, } \\
\text { Occup: white- } \\
\text { collar workers } \\
\text { (nonmanage- } \\
\text { ment position, } \\
\text { VDT workers } \\
\& \text { clerks), Organ: } \\
\text { printing and PC } \\
\text { manufacturing } \\
\text { company }\end{array}$ & $\begin{array}{l}\text { Longitudinal: } \\
\text { test of linear relation, } \\
\text { baseline 1990, } \\
\text { follow-up 1993 }\end{array}$ & $\begin{array}{l}\text { Con \& MLinRA, } \\
\text { Con: age or late } \\
\text { night meal }\end{array}$ & $\begin{array}{l}\text { Obj (time clocks), } \\
\text { OH }- \text { month during } \\
\text { 3-year study peri- } \\
\text { od, mean 45.5, } \\
\text { SD } 15.6\end{array}$ & $\begin{array}{l}\text { Physiology } \\
\text { Serum cholesterol } \\
\text { Serum triglycerides } \\
\\
\text { Behavior } \\
\text { BMl } 1993 \\
\text { Waist circumference } 1993 \\
\text { BMl change in } 3 \text { years } \\
\text { Waist circumference change } \\
\text { in } 3 \text { years } \\
\text { Skinfold thickness } \\
\text { Dinner time } \\
\text { Breakfast } \\
\text { Exercise } \\
\text { Snacking } \\
\text { Smoking } \\
\text { Alcohol consumption } \\
\text { Preference for rich, fatty } \\
\text { foods }\end{array}$ & $\begin{array}{l}0^{\text {uni }} \\
\text { Ouni }^{\text {uni }} \\
0^{\text {uni }} \\
0^{\text {uni }} \\
+^{\text {multi }} \\
+^{\text {multi }} \\
0^{\text {uni }} \\
+^{\text {uni }} \\
0^{\text {uni }} \\
0^{\text {uni }} \\
0^{\text {uni }} \\
0^{\text {uni }} \\
\text { Ouni }^{\text {uni }} \\
\text { 0uni }\end{array}$ \\
\hline $\begin{array}{l}\text { Nakanishi } \\
\text { et al, } 1999 \\
\text { (39) }\end{array}$ & $\begin{array}{l}\mathrm{N}=869 \text {, Japan, } \\
\text { males: } 100 \% \text {, } \\
\text { A }_{\mathrm{b}}: 35-54 \text { years, } \\
\text { Occup: office/ } \\
\text { white-collar } \\
\text { workers, Organ: } \\
\text { building contrac- } \\
\text { tors }\end{array}$ & $\begin{array}{l}\text { Longitudinal: } \\
\text { comparison of two } \\
\text { groups varying in } \\
\text { workhours at base } \\
\text { line, baseline } 1994 \text {, } \\
\text { follow-up } 1997\end{array}$ & $\begin{array}{l}\text { MLogRA, Con: } \\
\text { age, life-style } \\
\text { factors }\end{array}$ & $\begin{array}{l}\text { SRWH, WH/day } \\
\text { at baseline, Dich: } \\
<10, \geq 10\end{array}$ & $\begin{array}{l}\text { Disease } \\
\text { Development of hypertension } \\
\text { in 3-year period, single } \\
\text { measurement at annual } \\
\text { health examination } \\
\text { Behavior } \\
\text { Smoking } \\
\text { Alcohol consumption } \\
\text { BMl } \\
\text { Eating breakfast } \\
\text { Eating between meals } \\
\text { Nutritional balance } \\
\text { Physical exercise } \\
\text { Hours of sleep }\end{array}$ & $\begin{array}{c}0^{\text {multi }} \\
0^{\text {multi }} \\
\text {-multi } \\
0^{\text {multi }} \\
0^{\text {multi }} \\
0^{\text {multi }} \\
0_{\text {multi }}^{\text {multi }} \\
\text {-multi }\end{array}$ \\
\hline $\begin{array}{l}\text { Nakanishi } \\
\text { et al, } 2001 \\
\text { (40) }\end{array}$ & $\begin{array}{l}\mathrm{N}=941, \text { Japan, } \\
\text { males: } 100 \% \text {, } \\
\mathrm{A}_{\mathrm{b}} \mathrm{:} \text { : } 35-54 \text { years, } \\
\text { Occup: white } \\
\text { collar workers } \\
\text { not in shift } \\
\text { system, Organ: } \\
\text { building con- } \\
\text { tractor }\end{array}$ & $\begin{array}{l}\text { Longitudinal: } \\
\text { comparison of six } \\
\text { groups varying in } \\
\text { workhours at baseline, } \\
\text { baseline 1994, follow- } \\
\text { up } 1999\end{array}$ & $\begin{array}{l}\text { MLogRA, Con: } \\
\text { age, occupation, } \\
\text { position \& life-style } \\
\text { factors }\end{array}$ & $\begin{array}{l}\text { SRWH, WH/day } \\
\text { at baseline, Cat: } \\
<8,8.0-8.9,9.0- \\
9.9,10.0-10.9 \text {, } \\
\geq 11\end{array}$ & $\begin{array}{l}\text { Disease } \\
\text { Development of borderline } \\
\text { hypertension } \\
\text { Development of definite } \\
\text { hypertension (in 5-year } \\
\text { period) }\end{array}$ & _multi \\
\hline $\begin{array}{l}\text { Nylén et al, } \\
2001 \text { (41) }\end{array}$ & $\begin{array}{l}\mathrm{N}=20632 \text {, } \\
\text { Sweden, males: } \\
54 \%, \mathrm{~A}_{\mathrm{b}}: 15-47 \\
\text { years, Occup: } \\
\text { diverse, Organ: } \\
\text { diverse }\end{array}$ & $\begin{array}{l}\text { Longitudinal: } \\
\text { comparison of two } \\
\text { groups varying in } \\
\text { overtime hours } \\
\text { at baseline, } \\
\text { baseline } 1973, \\
\text { follow-up } 1996\end{array}$ & $\begin{array}{l}\text { MLoGR, SA: gender, } \\
\text { Con: demographic } \\
\text { variables, life-style } \\
\text { factors, extraversion \& } \\
\text { long lasting or serious } \\
\text { illness }\end{array}$ & $\begin{array}{l}\text { SRWH, OH/week } \\
\text { at baseline, } \\
\text { Dich: } \leq 5,>5\end{array}$ & $\begin{array}{l}\text { Mortality } \\
\text { Mortality from all causes: } \\
\text { 5-year follow-up (1973- } \\
\text { 1977) } \\
\text { 24-year follow-up (1973- } \\
\text { 1996) }\end{array}$ & $\begin{array}{l}t^{\text {multi }} \text { male, NR fe- } \\
\text { male } \\
\text {-multi male, }+^{\text {multi fe }} \\
\text { male }\end{array}$ \\
\hline
\end{tabular}


Table 1. Continued

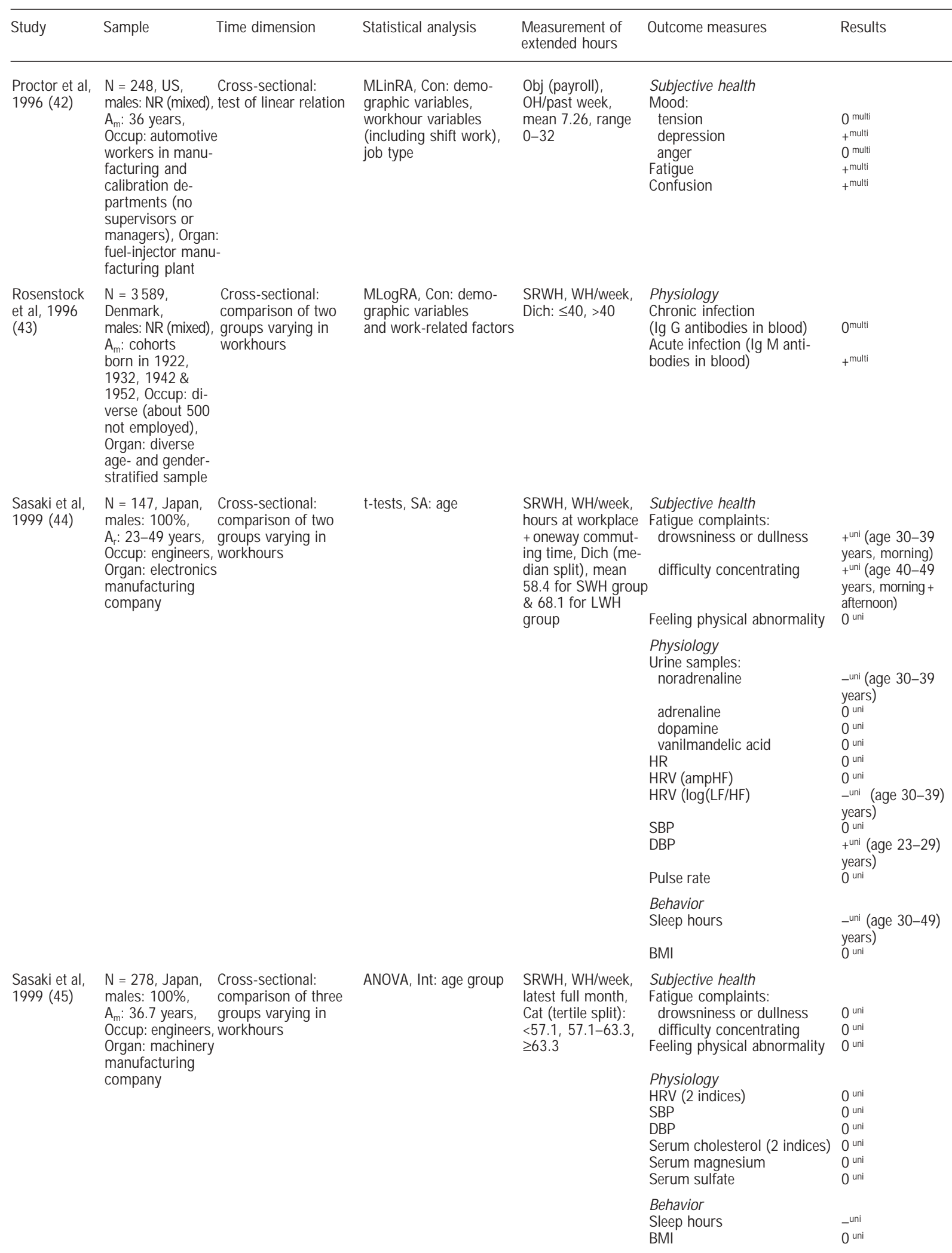


Table 1. Continued

\begin{tabular}{|c|c|c|c|c|c|c|}
\hline Study & Sample & Time dimension & Statistical analysis & $\begin{array}{l}\text { Measurement of } \\
\text { extended hours }\end{array}$ & Outcome measures & Results \\
\hline $\begin{array}{l}\text { Sokejima \& } \\
\text { Kagamimori, } \\
1998 \text { (46) }\end{array}$ & $\begin{array}{l}\mathrm{N}_{\text {case }}=195 \\
\mathrm{~N}_{\text {ref }}=331 \\
\text { Japan, males: } \\
\text { 100\%, A: } 30-69 \\
\text { years, Occup: } \\
\text { diverse, Organ: } \\
\text { diverse }\end{array}$ & $\begin{array}{l}\text { Case-referent: } \\
\text { cases: patients admitted } \\
\text { to hospital with acute } \\
\text { myocardial infarction, } \\
\text { referents: healthy, } \\
\text { matched for age and } \\
\text { occupation }\end{array}$ & $\begin{array}{l}\text { MLogRA, Con: demo- } \\
\text { graphic variables and } \\
\text { risk factors }\end{array}$ & $\begin{array}{l}\text { SRWH, (salary } \\
\text { overview gives } \\
\text { information), } \\
\text { WHdday, in month } \\
\text { before infarction } \\
\text { or recruitment, } \\
\text { Cat: } \leq 7,7-9,9-11 \text {, } \\
\geq 11\end{array}$ & $\begin{array}{l}\text { Disease } \\
\text { Acute myocardial infarction } \\
\text { needing hospital treatment }\end{array}$ & $t^{\text {multi }}$ \\
\hline $\begin{array}{l}\text { Steptoe et al, } \\
1998 \text { (47) }\end{array}$ & $\begin{array}{l}\mathrm{N}=71, \mathrm{UK} \\
\text { males: } 38 \%, \\
\mathrm{~A}_{\mathrm{m}}: 35.1 \text { years, } \\
\text { Occup: diverse, } \\
\text { Organ: large de } \\
\text { partment store }\end{array}$ & $\begin{array}{l}\text { Repeated measures: } \\
\text { comparison of busy } \\
\text { week and reference } \\
\text { week, } 4 \text { measure } \\
\text { ments over 6-month } \\
\text { period }\end{array}$ & $\begin{array}{l}\text { RMANOVA, Int: } \\
\text { gender, job strain } \\
\& \text { job social support } \\
\text { (median split) }\end{array}$ & $\begin{array}{l}\text { SRWH, WH/week } \\
\text { in past } 7 \text { days, } \\
\text { mean } 32.6-48.0^{d} \\
\text { in week with } \\
\text { shortest and } \\
\text { longest hours } \\
\text { respectively }\end{array}$ & $\begin{array}{l}\text { Subjective health } \\
\text { Perceived stress } \\
\text { Total GHQ } \\
\text { Physiology } \\
\text { Cortisol (saliva) } \\
\text { Behavior } \\
\text { Smoking behavior } \\
\text { Alcohol consumption }\end{array}$ & $\begin{array}{l}\text { 0ws } \\
+^{\text {ws female }} \\
+^{\text {ws }} \text { male }\end{array}$ \\
\hline $\begin{array}{l}\text { Trinkoff \& } \\
\text { Storr, } 1998 \\
\text { (48) }\end{array}$ & $\begin{array}{l}\mathrm{N}=3917, \mathrm{US}, \\
\text { males: } 5 \% \\
\mathrm{~A}_{\mathrm{m}}: 43 \text { years, } \\
\text { Occup: nurses, } \\
\text { Organ: diverse } \\
\text { (hospitals, clinics) }\end{array}$ & $\begin{array}{l}\text { Oross-sectional: } \\
\text { comparison of four } \\
\text { groups varying in } \\
\text { frequency of overtime } \\
\text { hours }\end{array}$ & ULogRA & $\begin{array}{l}\text { SRWH, OF: number } \\
\text { of days in past } \\
\text { month, Cat: } 0,1-3 \text {, } \\
4-7, \geq 8 \text { (=0-64 } \\
\text { hours/month) }\end{array}$ & $\begin{array}{l}\text { Behavior } \\
\text { Substance use in past year: } \\
\text { alcohol use } \geq 5 \text { drinks per } \\
\text { occasion } \\
\text { use of psychotropic drugs } \\
\text { smoking } \geq 10 \text { cigarettes/day) }\end{array}$ & $\begin{array}{l}+^{\text {uni }} \\
0^{\text {uni }} \\
0^{\text {uni }}\end{array}$ \\
\hline $\begin{array}{l}\text { Tyssen et al, } \\
2000 \text { (49) }\end{array}$ & $\begin{array}{l}\mathrm{N}=371, \\
\text { Norway, } \\
\text { males: } 44 \%, \\
\mathrm{~A}_{\mathrm{m}}: 29 \text { years, } \\
\text { Occup: junior } \\
\text { house officers, } \\
\text { Organ: hospitals }\end{array}$ & $\begin{array}{l}\text { Cross-sectional: } \\
\text { test of linear relation }\end{array}$ & $\begin{array}{l}\text { MLogRA, Con: demo- } \\
\text { graphic variables, } \\
\text { personality, stress \& } \\
\text { skills, work-related } \\
\text { factors }\end{array}$ & $\begin{array}{l}\text { SRWH, WH/week, } \\
\text { mean } 49.4, \mathrm{SD} 7.5, \\
\text { range } 30-88\end{array}$ & $\begin{array}{l}\text { Subjective health } \\
\text { Mental health problems need- } \\
\text { ing treatment }\end{array}$ & $0^{\text {multi }}$ \\
\hline $\begin{array}{l}\text { Tyssen et al, } \\
2001(50)\end{array}$ & $\begin{array}{l}\mathrm{N}=371, \\
\text { Norway, } \\
\text { males: } 44 \%, \\
\mathrm{~A}_{\mathrm{m}} \text { : } 29 \text { years, } \\
\text { Occup: junior } \\
\text { house officers, } \\
\text { Organ: hospitals }\end{array}$ & $\begin{array}{l}\text { Oross-sectional: } \\
\text { test of linear relation }\end{array}$ & $\begin{array}{l}\text { MLogRA, Con: demo- } \\
\text { graphic variables, } \\
\text { personality, life events, } \\
\text { job stress \& hours } \\
\text { asleep when on calls }\end{array}$ & $\begin{array}{l}\text { SRWH, WH/week, } \\
\text { mean } 49.4, \text { SD } 7.5 \\
\text { range } 30-88\end{array}$ & $\begin{array}{l}\text { Subjective health } \\
\text { Suicidal thoughts } \\
\text { Suicidal planning }\end{array}$ & Omulti $^{\text {multi }}$ \\
\hline $\begin{array}{l}\text { Voss et al, } \\
2001 \text { (51) }\end{array}$ & $\begin{array}{l}\mathrm{N}=2628, \\
\text { Sweden, } \\
\text { males: } 54 \%, \\
\text { A }_{\mathrm{m}}: 41 \text { years, } \\
\text { Occup: diverse, } \\
\text { Organ: postal } \\
\text { service }\end{array}$ & $\begin{array}{l}\text { Cross-sectional: } \\
\text { comparison of two } \\
\text { groups varying in } \\
\text { overtime hours }\end{array}$ & $\begin{array}{l}\text { MLogRA, SA: gender, } \\
\text { Con: work-related } \\
\text { factors }\end{array}$ & $\begin{array}{l}\text { SRWH, OH/year, } \\
\text { Dich: } \leq 50,>50\end{array}$ & $\begin{array}{l}\text { Work disability } \\
\text { Incidence of sickness } \\
\text { in } 1 \text { year [dichotomous: low } \\
\text { ( } 2 \text { events/year) and high } \\
\text { ( } \geq 2 \text { events/year), data from } \\
\text { company's register] }\end{array}$ & _multi \\
\hline $\begin{array}{l}\text { Yasuda et al, } \\
2001 \text { (52) }\end{array}$ & $\begin{array}{l}\mathrm{N}=142, \text { Japan, } \\
\text { males: } 100 \% \text {, } \\
\mathrm{A}_{\mathrm{m}}: 36.6 \text { years, } \\
\text { Occup: diverse, } \\
\text { Organ: technology } \\
\text { development } \\
\text { company }\end{array}$ & $\begin{array}{l}\text { Cross-sectional: } \\
\text { comparison of three } \\
\text { groups varying in } \\
\text { workhours } \\
y\end{array}$ & $\begin{array}{l}\text { MLinRA, Con: } \\
\text { sleep hours, } \\
\text { smoking \& age }\end{array}$ & $\begin{array}{l}\text { SRWH, WH/week, } \\
\text { hours at work- } \\
\text { place + oneway } \\
\text { commuting time, } \\
\text { Cat (tertile split): } \\
<55,<65, \geq 65 \text { hours }\end{array}$ & $\begin{array}{l}\text { Physiology } \\
\text { Immunologic parameters } \\
\text { in blood: } \\
\text { Thelper cells } \\
\text { T suppressor or killer cells } \\
\text { natural killer cells }\end{array}$ & $\begin{array}{c}0^{\text {multi }} \\
0^{\text {multi }} \\
\text {-multi e }\end{array}$ \\
\hline
\end{tabular}

a Results after correction in reply to comments by Epstein (53).

b Stronger relation for workhours and psychological strain for individuals low in self-efficacy.

${ }^{c}$ A short report about this study was published in 1997 (54).

d Mean workhours longer in first assessment period, but other period could have had longest hours for some participants.

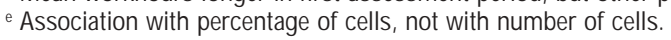

Statistical analysis. This column lists the statistical tests used for the association between extended hours and outcome variables and the covariates or confounders that were included in the analyses (demographic variables, work characteristics, home characteristics, personality, health-related behavior, physiological indices). Two studies $(30,47)$ reported within-subject tests and did not control for covariates. In within-subject designs, the participants served as their own controls, and confounding of extended hours with psychosocial job characteristics and other factors was less likely to occur. Five studies $(30,31,44,45,48)$ did not control for covariates and reported only univariate between-subject associations. Four studies $(26,28,33,38)$ reported 
univariate tests for some associations and multivariate tests for others. The remaining 17 studies $(27,29,32$, 34-37, 39-43, 46, 49-52) reported multivariate associations for all the outcome variables. In some studies, the relationship between extended hours and the outcome variables was studied in specific subgroups of participants (eg, based on gender, age, or type of occupation), or moderator effects were studied with interaction terms. Three studies tested moderating effects of gender (41, $47,51)$, and two studies investigated moderating effects of age group $(31,45)$. Other moderators tested were hypertension status (30), self-efficacy and collective efficacy (32), and job strain and social support (47).

Measurement of extended hours. Extended workhours could be measured as overtime hours (per day, week, month, or year), or the total number of workhours (per day, week, or month). Only two studies $(38,42)$ used officially registered workhours as a source of information; the others relied on self-reports.

Outcome measures. Some studies included more than one category of outcome measure. Only 1 study investigated mortality (41), 4 independent studies (28, 35, 39, $40,46)$ investigated diagnosed disease, 2 studies investigated work disability $(36,51), 13$ studies $(26,27$, 29-32, 42, 44, 45, 47, 49, 50) investigated subjective health, 12 studies $(30,31,33,37,38,40,43-45,47,52)$ investigated physiological outcome measures, and 12 studies $(26,28,30,33,34,38,39,44,45,47,48)$ investigated behavioral outcome measures.

Results. For every outcome variable, the results column lists whether it had a significant negative, significant positive, or no significant relationship with the measure of extended hours. If there was a positive or negative association, it is noted whether it was a univariate association (only reported if the association was not tested by means of a multivariate analysis technique), a multivariate association (after correction for covariates) or a within-subject association. If a relationship was significant only for a specific subgroup of participants (separate analysis or interaction term), this fact is also indicated in the results column.

\section{Results}

Table 2 summarizes the results of the 27 selected studies. Within each category of outcome measures, subcategories of similar outcome measures were distinguished. For each subcategory of outcome measures, table 2 indicates how many independent studies included this outcome measure. If more than half or all the studies that included a particular outcome measure found an association with long workhours, the direction of the association (+ or - ) is given in the last column of the table. If the association was found in more than one study and all studies that found an association corrected for covariates, a double plus (++) or a double minus $(--)$ is used to indicate that the evidence for this association was particularly strong. If only half of the studies or fewer found an association, the direction (positive or negative) of the association is given between brackets. If some studies found a positive association and others found a negative association, the mixed evidence is indicated with a \pm sign. If none of the studies that included the outcome variable found an effect, a zero is used.

\section{Mortality}

Only one study (41) investigated the association between extended workhours and mortality. Working overtime more than 5 hours a week at baseline was associated

Table 2. Overview of the strength of the evidence for the associations between long workhours and several health-related outcome variables. For further explanation see the text. $(\mathrm{RSA}=$ respiratory sinus arrhythmia, LF = low frequency, HF = high frequency)

\begin{tabular}{|c|c|c|}
\hline Category & $\begin{array}{l}\text { Independent } \\
\text { tests of } \\
\text { association } \\
\text { (N) }\end{array}$ & $\begin{array}{l}\text { Summarized } \\
\text { evidence for } \\
\text { association }\end{array}$ \\
\hline \multicolumn{3}{|l|}{ Mortality } \\
\hline All-cause & 1 & \pm \\
\hline \multicolumn{3}{|l|}{ Disease } \\
\hline Cardiovascular disease & 2 & + \\
\hline Hypertension & 1 & - \\
\hline Diabetes & 1 & + \\
\hline \multicolumn{3}{|l|}{ Work disability } \\
\hline Disability retirement & 1 & + \\
\hline Sickness absence & 1 & - \\
\hline \multicolumn{3}{|l|}{ Subjective health } \\
\hline General ill health & 6 & {$[+]$} \\
\hline Psychological ill health & 3 & \pm \\
\hline Physical ill health & 2 & ++ \\
\hline Fatigue & 6 & + \\
\hline \multicolumn{3}{|l|}{ Physiology } \\
\hline Heart rate & 3 & {$[+]$} \\
\hline Heart rate variability (RSA) & 4 & {$[-]$} \\
\hline Heart rate variability (LF/HF) & 3 & \pm \\
\hline Blood pressure & 5 & {$[+]$} \\
\hline Immunity & 2 & -- \\
\hline Cholesterol & 4 & {$[-]$} \\
\hline Fasting blood sugar & 1 & + \\
\hline Noradrenaline & 1 & - \\
\hline \multicolumn{3}{|l|}{ Behavior } \\
\hline Sleep hours & 7 & - \\
\hline Alcohol consumption & 6 & {$[ \pm]$} \\
\hline Smoking & 7 & {$[+]$} \\
\hline Use of psychotropic drugs & 1 & 0 \\
\hline Unhealthy eating habits & 2 & {$[+]$} \\
\hline Exercise & 3 & 0 \\
\hline Body mass index or obesity & 6 & {$[ \pm]$} \\
\hline
\end{tabular}


with higher all-cause mortality after 5 years for men [relative risk (RR) 2.00], but no association could be reported for women because there were no exposed cases (apparently, no women who worked overtime had died). After a follow-up period of 24(!) years, working overtime more than 5 hours a week at baseline was associated with higher mortality for women $(\mathrm{RR}=1.92)$, but not for men, and working overtime less than 5 hours a week was associated with lower mortality for men ( $R R=0.58)$. Thus this study provided mixed evidence for a relationship between overtime work and mortality from all causes.

\section{Diagnosed disease}

Cardiovascular disease. Two studies $(28,46)$ addressed the relationship between long workhours and cardiovascular disease in case-referent designs using all-male samples. Emdad et al (28) showed that patients with ischemic heart disease have longer workhours ( 8.5 hour/ day) than hypertensive (6.3 hours/day) and normotensive (6.5 hours/day) referents. Sokejima \& Kagamimori (46) found that working more than 11 hours a day was associated with increased risk $(R R=2.94)$ of myocardial infarction, after control for covariates. Interestingly, working 7 hours or less per day was also associated with an increased risk of acute myocardial infarction $(\mathrm{RR}=2.83)$.

Hypertension. Two studies $(39,40)$ concerning the same all-male study population found that long workhours were associated with a reduced risk of developing hypertension. Nakanishi et al (39) showed that, over a 3year period, those who worked 10 hours or more a day had an increased risk of developing hypertension $(\mathrm{RR}=0.58)$. After a 5 -year period (40), the risk of both developing borderline hypertension $(\mathrm{RR}=0.48)$ and definite hypertension $(\mathrm{RR}=0.33)$ was increased for those who worked 11 hours or more a day, after correction for covariates.

Diabetes. The only other disease that was studied in relation to extended hours was diabetes (35). This study revealed an increased risk of noninsulin-dependent diabetes mellitus $(\mathrm{RR}=3.73)$ for those who worked more than 50 hours of overtime a month in an all-male sample after correction for covariates.

\section{Work disability}

Two studies $(36,51)$ addressed work disability. After correction for covariates, working 60 hours or more a week was found to be associated with an increased risk of disability retirement (36) $(\mathrm{RR}=2.75)$, and working more than 50 hours of overtime per year was associated with lower sickness absence (51), both for women $(\mathrm{RR}=0.58)$ and for men $(\mathrm{RR}=0.70)$.

\section{Subjective health}

General health. Six studies $(26,27,29,32,47,49)$ investigated the association between overtime work and general subjective health. In one of these studies (29), the participants were asked whether their job had a positive or a negative impact on their psychological and physical health. The impact could be positive for both indicators, negative for both indicators, or mixed. Working more than 45 hours a week was found to be associated with more unambiguously negative effects of work on health $(R R=1.24)$. In two other studies $(26,47)$ the total score of the 28 -item version of the General Health Questionnaire was used as a health indicator. In neither of these studies was the total score associated with extended hours. In three studies $(27,32,49)$ other general health indicators were used. Two of these studies (27, 49) showed no association between extended workhours and health indicators, whereas the third (32) showed that extended workhours were associated with adverse general health.

Psychological health. Three studies $(26,42,50)$ focused on specific indicators of psychological health. One (26) included subscales of the General Health Questionnaire, which focused on psychological health (ie, social dysfunction and severe depression). Extended workhours were associated with social dysfunction, but depression was not associated with extended hours. Only one other study included depression as an outcome variable (42), and it found that extended hours were associated with higher levels of depression. This study also included other specific indicators of psychological health and found no association between extended hours and tension or anger, but confusion was higher for those who worked more overtime hours. Finally, one study focused on suicidal tendency (50), which can be regarded as a severe depression-related outcome. In this study, longer workhours were found to be associated with fewer suicidal thoughts, and there was no association between extended workhours and suicidal planning.

Physical health. Two studies $(26,32)$ investigated the relationship between self-reported physical health (somatic or psychosomatic symptoms) and extended workhours. One of these (26) found a positive association between long workhours and somatic symptoms, but not between long workhours and psychosomatic symptoms or physical ailments during the last year. The other (32) reported a positive association between extended workhours and physical strain. 
Fatigue. A total of six studies $(30,31,42,44,45)$ included measures of fatigue. Five of these $(30,31,44$, 45 ) used the fatigue symptoms scale that was developed by the Japanese Association of Industrial Health. In some, a distinction was made between three types of symptoms, and in some the questionnaire was filled out both before and after work. Four of these five studies found a positive association between extended workhours and fatigue, although this relationship was sometimes only valid for particular subgroups of participants. One additional study (42) also found a positive association between extended hours and another measure of fatigue.

\section{Physiological measures}

Cardiovascular indices. Heart rate, heart rate variability, and related measures were included in six studies (30, $31,33,44,45)$. Single measurements of heart rate and pulse (44) and 24-hour registration (30) of heart rate showed no association with extended hours between subjects. Mean 24-hour heart rate was significantly higher in a long workhour period ( 74 beats/minute) than in a relatively short workhour period ( 69 beats/minute) in a within-subject study (30). Respiratory sinus arrhythmia (heart rate variability with a frequency between 0.15 and $0.30 \mathrm{~Hz}$, used as an indicator of activity of the vagus nerve or the parasympathetic nervous system) was included in four studies $(31,33,44,45)$. Only one (33) found lower respiratory sinus arrhythmia during standing rest for those who worked extended hours (but not while lying down and only for one of the two indicators of the disorder); the other studies found no relationship. Three studies $(33,44,45)$ included the ratio between low-frequency and high-frequency components of heart rate variability, which is used as an indicator of sympathovagal balance. In one study (33), this indicator was found to be higher for those who work extended hours, but only during standing rest and not during lying down. The other two studies found no relationship (45) or a negative association for a specific age group (44). Mayer wave-related sinus arrhythmia (heart rate variability with a frequency between 0.05 and $0.15 \mathrm{~Hz}$, used as a combined indicator of sympathetic and vagal activity) was included in one study (33), but it was not associated with extended workhours. Blood pressure was used as an outcome measure in five studies $(30,31,44,45)$. Extended workhours were not associated with single measurements of systolic and diastolic blood pressure except for some associations in specific age groups. The two studies that included 24-hour registrations of blood pressure showed significant associations, however. Hayashi et al (30), in their cross-sectional study, showed that systolic blood pressure was significantly higher $(125 \mathrm{~mm} \mathrm{Hg})$ among normotensives who worked an average of 60 hours of overtime a month than it was in referents (117 $\mathrm{mm} \mathrm{Hg}$ ) who worked an average of 30 overtime hours per month. Their repeated-measures study (30) had a within-subject design and revealed that, in a busy period (96 overtime hours per month), both systolic blood pressure (127 $\mathrm{mm} \mathrm{Hg}$ ) and diastolic blood pressure (83 $\mathrm{mm} \mathrm{Hg}$ ) were higher than in the reference period (121 $\mathrm{mm} \mathrm{Hg}$ and $79 \mathrm{~mm} \mathrm{Hg}$, respectively).

Immunologic indices. Two studies $(43,52)$ focused on immunologic indices. One study (43) investigated the relationship between extended workhours and immunoglobulin $\mathrm{G}$ and immunoglobulin $\mathrm{M}$, indicators of chronic and acute Heliobacter pylori infection, respectively. Heliobacter pylori infection is thought to be a predictor of peptic ulcer disease. Working more than 40 hours a week was related to an increased risk of acute infection $(\mathrm{RR}=2.0)$, but not with risk of chronic infection. The other study (52) investigated the relationship between workhours and other types of immunologic indices and revealed no association with $\mathrm{T}$ helper and $\mathrm{T}$ suppressor cells. However, there was a negative association between extended workhours and the percentage of natural killer cells. This finding suggests a deterioration of immunity for those who work long hours.

Ather biochemical indices. Many different biochemical indices derived from blood, urine, and saliva samples have been studied in relation to extended hours, but only cholesterol has been included in more than one study $(30,31,38,45)$. None of the studies found an association between extended workhours and cholesterol levels for the whole study population, but in one (31) those who worked extended hours were found to have a lower cholesterol level in a specific age group (40-49 years). A large number of other biochemical indicators were measured in only one study. Significant associations with extended workhours were only found for two measures. Fasting blood sugar was found to be relatively high in normotensives who worked extended hours (30). A negative association between noradrenaline levels in urine and extended hours was found in a specific age group (44), and this finding indicates decreased activity of the sympathetic nervous system. One study (37) investigated the relationship between extended hours and urinary carcinogens or mutagens. Extended workhours were expected to affect the duration of exposure to carcinogens, but no significant associations were found. Other biochemical indicators that were included in only one study were blood fructosamine (blood sugar), urea nitrogen (kidney function) and creatinine (kidney function) (30), blood triglycerides (plasma lipids/fat, associated with diabetes mellitus) (38), 
urinary adrenaline, dopamine and vanilmandelic acid (stress hormones) (44), cortisol (47), and serum magnesium (kidney function) and sulfate (cardiovascular disease) (45). None of these indices were associated with extended workhours.

\section{Health-related behavior}

Sleep hours. Seven studies $(30,33,34,39,44,45)$ investigated associations between extended workhours and hours of sleep. All the studies (except for reference 33 ) found negative associations between sleep hours on weekdays and extended workhours. Kageyama et al (33) reported a univariate association between long workhours and longer sleep hours before holidays. Furthermore, those who worked longer hours appeared to have a longer sleep debt (the difference between required sleep hours and actual sleep hours) than those with normal workhours (34).

Alcohol consumption. The association between extended workhours and alcohol consumption was investigated in six studies $(26,30,38,39,47,48)$. The evidence was mixed. One study (26) found a negative association between extended workhours and alcohol consumption, three studies $(30,38,39)$ found no association, and two studies found a positive association $(47,48)$, in one case only for men. Some studies focused on the frequency of alcohol consumption, whereas others focused on the amount of alcohol consumed per occasion. Frequency of alcohol consumption (or a habit of drinking) was not associated with long workhours $(30,38,39)$. The amount of alcohol consumed per occasion or in the past week was negatively associated with extended hours in one study (26), but the association was positive in two others $(47,48)$.

Smoking. Associations between extended workhours and smoking were tested in seven studies (28, 30, 38, $39,47,48)$. Smoking behavior was sometimes assessed dichotomously (yes; no), and sometimes as the number of cigarettes smoked a day. One study (47) found that women smoked more cigarettes in busy weeks with long workhours (within-subject effect). The other six studies did not show significant associations.

Psychotropic drugs. Extended workhours were not associated with the use of psychotropic drugs (48).

Eating habits. Two studies $(38,39)$ provided information about the relationship between extended hours and eating habits. Eating behavior was investigated with several indicators. Eating breakfast and eating between meals (or snacking) were included in both studies and not associated with extended workhours. All other indicators were included in one study only. Not surprisingly, dinnertime was later for people who worked longer hours (48). Eating a nutritionally balanced diet, salt intake, and a preference for rich, fatty foods were not associated with extended workhours.

Exercise. Physical exercise was included in three studies $(33,38,39)$, and it was not associated with extended workhours.

Body mass index or obesity. Six studies (28, 30, 38, 39, $44,45)$ investigated the relationship between extended hours and body mass index (BMI), sometimes supplemented with other indicators of obesity. Two studies found a significant association, one positive (28) and one negative (39). Another study (38) found that BMI increases in a 3-year period were positively associated with extended workhours, but the increase could also be explained by later dinner times. Skinfold thickness was not associated with extended hours (38).

\section{Discussion}

\section{Recent evidence for the association between long workhours and health}

The first research question was whether recent literature shows evidence of a relationship between long workhours and specific aspects of health. Four categories of health outcomes were distinguished, and within these categories a further differentiation was made into several subcategories of highly similar outcome measures. The first category of health outcomes was mortality. The only study that addressed mortality found differential effects for women (higher all-cause mortality after 24 years of follow-up) and men (higher mortality after 5 years of follow-up but not after 24 years). Possible explanations for these effects could be that the men and women probably had different types of jobs or that the men had changed jobs and work habits more than the women had. Although the study controlled for covariates, work characteristics were not included as possible confounders. Alternatively, the so-called "double load" of women-the fact the many women combine their jobs with child care responsibilities (55)_-may be an explanation for these results. Nevertheless, it can be doubted whether working overtime 24 years ago is a sensible predictor of mortality.

The second category of outcome measures was diagnosed disease. Traditionally, research on psychological factors in occupational health has devoted a lot of attention to cardiovascular disease. This review includes 
two recent studies concerning the development of cardiovascular disease and one study concerning the development of hypertension. Long workhours were associated with an increased risk of cardiovascular disease but a decreased risk of hypertension. This is a surprising result because hypertension is regarded as an important risk factor for cardiovascular disease. A clue to the interpretation of these results may be that, in the hypertension study, BMI was negatively associated with extended hours, whereas there was a positive association for the professional drivers in one of the cardiovascular disease studies. This pattern of results suggests that the relationship between overtime work and cardiovascular disease may depend on changing eating patterns and physical exercise. The only other disease that was studied in relation to long workhours was noninsulin-dependent diabetes mellitus. This type of diabetes is often accompanied by high blood pressure. The risk of developing diabetes was increased for those who worked long hours. This is an important finding because earlier research has concentrated on cardiovascular disease and studies involving other diseases were virtually nonexistent.

Work disability was the third category of outcome variable. Working long hours was associated with increased risk of disability retirement and decreased sickness absence. These apparently contradictory results may be due to differences in exposure. The study on disability retirement included persons who worked more than 60 hours a week, whereas the study on sickness absence focused on moderate amounts of overtime (more than 50 hours a year). Alternatively, persons who work long hours may be very committed to their jobs and not tend to report in sick for minor health complaints, which could develop into serious health problems and eventually to disability. Long workhours also imply that workers are exposed to work hazards for a longer period of time, and therefore the risk, for example, of becoming involved in a serious accident increases and, as a consequence, so does the risk of becoming disabled. Both disability retirement and sickness absence are measures that are influenced not only by health, but also by many other factors. Therefore, associations between these variables and workhours should be interpreted with caution.

The last category of health outcomes was subjective health. The association between workhours and general subjective health was not strongly supported, but the effects that were found were in the expected direction. Long workhours appeared to be associated with impaired physical health and fatigue. Regarding psychological health, long workhours were associated with social dysfunction and confusion, but associations with depression-related outcomes were mixed. In general, the results suggest that specific indicators of subjective health are more clearly associated with long workhours than broad indicators.

In summary, most studies found either no association between long workhours and adverse health or an association in the expected direction (ie, long workhours in association with adverse health). These results show that there is good reason to be concerned about the possible detrimental effects of long workhours on health, in particular cardiovascular disease, diabetes, illnesses leading to disability retirement, subjectively reported physical ill health, and subjective fatigue. However, reverse or counterintuitive associations were found for the development of hypertension and sickness absence. It can be concluded that there is evidence of a link between long workhours and ill health, but there is a serious shortage of well-controlled studies that can confirm and strengthen the evidence.

\section{Recent evidence for the association between long workhours and physiological change}

The second research question was whether recent literature provides evidence of physiological changes in those who work long hours. Studies on associations between long workhours and cardiovascular indices were the most common. The evidence found for cardiovascular changes was rather weak. Only a few studies found effects, but the studies that did find significant associations applied within-subject designs or controlled for covariates. For heart rate and blood pressure, associations in the expected direction were found: long workhours in association (in some studies) with a higher heart rate and a higher blood pressure. Sustained increases in heart rate and blood pressure are thought to be associated with cardiovascular disease. The results with respect to heart rate variability were inconsistent. The results of a study that controlled for several covariates pointed at a sympathodominant state. In that case, the line of reasoning would be that chronic stress leads to increased activity of the sympathetic nervous system. A sympathodominant state is thought to be associated with increased risk of cardiovascular disease. However, another study found evidence for vagal dominance in those who work long hours (although only for a specific age group and without control for covariates), and this evidence was supported by decreased noradrenaline levels. The line of reasoning in that study was that chronic sleep deprivation leads to increased vagal activity.

The strongest evidence within this category of outcome measures was found for a relationship between long workhours and depression of the immune system. This is a very important result because Spurgeon and her co-workers (4) stated that no studies on immunologic effects of long workhours were known at that time. 
Nevertheless, even now, the information that is available is based on only two well-controlled studies that investigated different immunologic indices (one measured immunologic parameters directly, whereas the other focused on a bacterial infection). Thus it remains to be seen whether long workhours are associated with specific immunologic changes and whether the association holds under varying circumstances. For example, a recent study (56) focused on taxi drivers who did and did not work overtime in two different years. This study showed that, during the economic depression in Japan, taxi drivers who worked extended hours had better immune responses than those who worked normal hours. The line of reasoning in the article was that taxi drivers who did not work overtime suffered from stress due to insufficient earnings. Thus the association between long workhours and immunity may not be as straightforward as it seems.

A large number of biochemical indices were studied in relation to long workhours, but only cholesterol was included as an outcome measure in more than one study. There was some very weak evidence that cholesterol levels may be lower for those who work long hours. This possibility is surprising because a high cholesterol level is regarded as an important risk factor for the development of cardiovascular disease. Furthermore, there was some evidence that fasting blood sugar was increased in persons who work long hours. This result is in line with the increased risk of noninsulin-dependent diabetes mellitus. Other biochemical indices were only included in one study, and no significant associations with long workhours were found.

In summary, there is some evidence for a lack of physiological recovery among those who work long hours. In particular, results point at cardiovascular changes (increased heart rate and blood pressure). Furthermore, there is evidence for an association between long workhours and reduced immunity. Thus long workhours are, at least under some circumstances, associated with a disturbance of vital physiological processes that are associated with an increased risk of development of cardiovascular disease, diabetes, and vulnerability to bacterial and viral infections. However, the evidence is inconclusive because many studies did not find significant effects and most studies did not control for covariates.

\section{Recent evidence for the association between long workhours and behavioral changes}

The third research question was whether the recent literature contains evidence that long workhours are associated with changes in health-related behavior. The relationship that was most consistently found was that long workhours are associated with short sleep hours. Sleep deprivation is known to be associated with decreased alertness, deterioration of performance, and affective changes (10). The association between long workhours and short sleep hours suggests that a lack of recovery may be the most important pathway that links extended workhours and health. It is unclear, however, whether these short sleep hours are caused by sleep problems - not being able to fall asleep or waking up during the night or early in the morning (57) —or whether the time spent in bed is simply shorter. In the first case, sleep loss or sleep debt could reflect a lack of physiological relaxation (sympathetic dominance), whereas in the second case, lack of sleep could cause vagal dominance, accompanied by feelings of drowsiness and fatigue. On the basis of the studies reviewed, it can be speculated that sympathetic dominance occurs in cases of acute or temporary sleep deprivation, and vagal dominance occurs in cases of chronic sleep deprivation. However, well-controlled longitudinal studies are necessary in order to see whether this explanation of the findings holds.

The use of three health-threatening substances (alcohol, tobacco, and psychotropic drugs) was studied in relation to long workhours. The evidence for an association between long workhours and alcohol consumption was mixed. The frequency of alcohol consumption was not associated with long workhours, but, in some studies, the amount of alcohol consumed per week or per occasion was. However, the association could be negative or positive. The workhours in the study in which the negative association was found were extremely long (the participants were junior doctors). Thus the participants probably did not have a chance to drink much alcohol because they were on duty most of the time. The number of cigarettes smoked was increased for women when they worked long hours (within-subject study), but this effect was not confirmed in other (between-subject) studies. The use of psychotropic drugs was only included as an outcome variable in a single study, and it was not associated with long workhours. Taken together, the evidence for changes in substance use for those who work long hours is not very strong. Nevertheless, substance use, in particular alcohol consumption and the intake of psychotropic drugs, may not only be a threat to health, but may also affect a worker's social life in a negative way, and make a worker more likely to become involved in accidents (at work or elsewhere). Therefore, a further investigation of this topic seems justified.

Three indicators were used to investigate the relationship between long workhours and the maintenance of a healthy body weight: eating behavior, exercise, and obesity. The third indicator can be seen as the result of (among others) the first two. There was hardly any evidence that long workhours are associated with unhealthy 
eating behavior, except for a late dinnertime, but it can be doubted whether this habit is unhealthy. No association with physical exercise was found. Nevertheless, some associations between long workhours and obesity were found in relatively well-controlled studies. In one study concerning professional drivers, long workhours were associated with a higher BMI, whereas in another study concerning white-collar workers, the opposite was found. This contradiction may be explained by a difference in the nature of work in both studies. Although white-collar work may not be particularly physically demanding, it certainly provides more opportunities for walking around and being physically active than driving. Thus it can be speculated that workers in sedentary jobs are likely to have a relatively high BMI when they work long hours.

In conclusion, there is some evidence for changes in behavioral life-style for those who work long hours. Those who work long hours seem to have short sleep hours (although this was only studied in samples with extremely long hours). This finding is in agreement with the disturbance of physiological processes already discussed, and it strengthens the evidence for the physiological recovery mechanism. In some circumstances, long workhours seem to be associated with increased smoking, alcohol consumption, and a higher BMI. However, only a small proportion of the studies found significant relationships between long workhours and health-related behavior, some reverse associations were found, and most of the studies did not control for covariates. Thus the evidence for the behavioral life-style mechanism that may explain the relationship between long workhours and ill health is rather weak.

\section{Gaps in the current literature and recommendations for future research}

Systematic reviews combine results from multiple studies in order to provide a comprehensive coverage of the evidence, but they also have some drawbacks (58-61). First, one of the risks of literature reviews is that some studies are not retrieved because the key words that guide the search are not mentioned in the title or the abstract of the article. Especially studies in which working long hours is only one of many predictors for a particular health-related outcome are likely to be missed. Therefore, the list of studies that were included in the review is probably incomplete. Second, due to publication bias, some studies that did not find significant associations between long workhours and health-related outcomes were probably not published. Publication bias leads to an overestimation of the threat that long workhours pose to health. Third, this review focused only on the presence of an association between long workhours and outcome variables, and the direction of that association. The size of the effects was not taken into account. Especially in large-scale studies with large samples, significant associations may sometimes not be very relevant. This situation would also imply that the possible effects of long workhours on health are overestimated. Despite these limitations, this review should provide a representative overview of the current knowledge concerning the possible health effects of long workhours, and it provides an opportunity to locate the main conceptual gaps in the literature and the methodological shortcomings of existing studies that can guide future research.

The review covered a period of 5.5 years and employed strict selection criteria. Nevertheless, the number of studies retrieved was about equal to the number of studies reviewed by Sparks and her colleagues (3), although the latter review concerned all studies published until 1996. Therefore, the association between long workhours and health-related outcomes has received much attention in the past few years. In their 1997 literature review, Spurgeon and her colleagues (4) concluded that there were three important gaps in the literature. First, the range of health indicators that had been used as outcome variables was limited to subjective health and cardiovascular disorders. Other disease-related outcome variables such as gastrointestinal disorders, musculoskeletal disorders, and problems associated with depression of the immune system had hardly received any attention. My review shows that the range of diagnosed diseases that have been studied in relation to long workhours is still rather limited, although a study on immunity (52) and a study concerning bacterial infection related to gastrointestinal disorders (43) were retrieved. In addition, it should be mentioned that two recent studies on musculoskeletal disorders $(62,63)$ hardly found any associations between complaints and working overtime (these studies were not included in this review because they did not measure extended workhours in enough detail). Second, most studies focused on workweeks of over 50 hours, and few studies addressed "moderate" overtime. Some of the studies in the review concerned moderate overtime (ie, most of the participants worked between 40 and 50 hours a week) and found significant associations with the outcome variables $(29,41,42,43,47,51)$. However, the outcome variables in these studies were so various that it was not possible to draw any general conclusions. A large proportion of the studies, particularly the Japanese studies, involved extremely long workhours. In many cases the reference groups (ie, the workers with relatively short workhours) worked more than 40 hours a week. Therefore, one cannot be certain that these workers did not already suffer from health complaints due to long workhours. The third limitation was that little was known about the factors that may moderate the 
relationship between long workhours and health. The studies that were included in the review hardly included any potential moderators. Apart from gender and age, the only moderators that were studied were self-efficacy and collective efficacy (32) and job strain and job social support (47). Thus little is known about the factors that may change the relationship between long workhours and health. It can be concluded that, to date, the gaps in the literature that were identified by Spurgeon and her colleagues (4) have not been filled.

The review of studies that include physiological indices and health-related behavior as outcome variables showed that the range of different outcomes that have been studied is rather broad. However, one topic that has hardly received any attention is the relationship between long workhours and hormone excretion, in particular adrenaline, noradrenaline, and cortisol. Some earlier research suggests that disturbances in stress hormone excretion may occur in long workhour situations or in high workload situations in general $(19,64,65)$. Some recent studies suggest that changes in hormonal reactivity are associated with a lack of recovery and exhaustion $(66,67)$. It seems particularly important to study the associations between long workhours, short sleep hours, and hormone excretion in future research, as it could provide a crucial causal link in the relationship between long workhours and cardiovascular and immunologic health problems.

The studies in the review had several methodological gaps. First, as already presented, most of the associations that were found need to be replicated in studies that control for possible confounders in order to be able to draw more certain conclusions. In particular, demographic variables, work characteristics, home characteristics, and personality factors should be included as covariates. Second, a large proportion of the studies included in the review was carried out with all-male samples. It could very well be that the outcomes associated with long workhours differ for men and women. On one hand, physiological changes related to long workhours may not be the same for men and women. Indeed, some studies suggest that cardiovascular responses to stress differ in men from those in women (68), and there are indications of differences in hormonal responses (64, 69). On the other hand, stress-related changes in healthrelated behavior, such as substance abuse, seem to differ between men and women $(47,70)$. Therefore, future research should investigate whether the associations between workhours and health-related outcomes that have been found for men can be confirmed in mixed-gender or all-female samples.

The third methodological flaw is that most of the studies included in the review were cross-sectional. Moreover, the longitudinal designs that were used were suboptimal because they usually used workhours at the baseline as a predictor of health complaints at the follow-up some years later. As the follow-up period becomes longer, participants are more likely to have, for example, changed jobs, and the predictive power of extended workhours at baseline is likely to decrease. One study (35) reported a correlation of 0.35 between overtime hours in two succeeding years. Future longitudinal studies should measure all variables (ie, independent and dependent variables) at all measurement points and test whether changes in workhours are associated with changes in health-related variables. Furthermore, in some types of jobs overtime work is seasonal (eg, accountancy, agriculture) and therefore provides the opportunity to make within-subject comparisons of outcome variables in long workhour and short workhour periods. Ideally, a group of workers in these types of jobs should be followed from day to day for a prolonged period of time in order to collect information about the possible accumulation of health complaints and changes in physiological indices and health-related behavior. These "natural experiments" could provide rather strong evidence for the existence or nonexistence of associations between long workhours and health-related outcomes.

\section{Acknowledgments}

The author would like to thank Michiel Kompier and Toon Taris for their comments on earlier drafts of this paper.

\section{References}

1. Cooper CL. Working hours and health[editorial]. Work Stress 1996;10:1-4.

2. Harrington JM. Working long hours and health. BMJ 1994;308:1581-2.

3. Sparks K, Cooper C, Fried Y, Shirom A. The effects of hours of work on health: a meta-analytic review. J Occup Org Psychol 1997;70:391-408.

4. Spurgeon A, Harrington JM, Cooper CL. Health and safety problems associated with long working hours: a review of the current position. Occup Environ Med 1997;54:367-75.

5. Åkerstedt T. Work hours, sleepiness and the underlying mechanisms. J Sleep Res 1995;4 Suppl 2:15-22.

6. Knauth P. Hours of work. In: Stellmann JM, editor. Encyclopedia of occupational health and safety. 4th ed. Geneva: International Labour Office; 1998. p 43.1-43.15.

7. Rosa RR. Extended workshifts and excessive fatigue. J Sleep Res 1995;4(2):51-6.

8. Smith L, Folkard S, Tucker P, Macdonald I. Work shift duration: a review comparing eight hour and 12 hour shift systems. Occup Environ Med 1998;55:217-29.

9. Thierry H, Meijman T. Time and behavior at work. In: Trian- 
dus H, Dunette, MD, Hough LM, editors. Handbook of industrial and organizational psychology; vol 4. 2nd ed. Palo Alto (CA): Consulting Psychologists Press; 1994. p 341-413.

10. Krueger GP. Sustained work, fatigue, sleep loss and performance: a review of the issues. Work Stress 1989;3:129-41.

11. Spurgeon A, Harrington JM. Work performance and health of junior hospital doctors: a review of the literature. Work Stress 1989;3:117-28.

12. Haratani T. Karoshi: death from overwork. In: Stellmann JM, editor. Encyclopedia of occupational health and safety. 4th ed. Geneva: International Labour Office; 1998. p 5.18-5.19.

13. Uehata T. Long working hours and occupational stress-related cardiovascular attacks among middle-aged workers in Japan. J Hum Ergol 1991;20:147-53.

14. Karasek RA. Job demands, job decision latitude, and mental strain: implications for job redesign. Admin Sci Q 1979;24: 285-308.

15. Karasek R. Control in the workplace and its health-related aspects. In: Sauter SL, Hurrell JJ, Cooper CL, editors. Job control and worker health. New York (NY): John Wiley; 1989. p 129-59.

16. Karasek R. Demand/control model: a social, emotional, and physiological approach to stress risk and active behaviour development. In: Stellmann JM, editor. Encyclopedia of occupational health and safety. 4th ed. Geneva: International Labour Office; 1998. p 34.6-34.14.

17. Meijman TF, Mulder G. Psychological aspects of workload. In: Drenth PJD, Thierry H, De Wolff CJ, editors. Handbook of work and organizational psychology; vol 2 (Work psychology). 2nd ed. Hove (UK): Psychology Press; 1998. p 5-33.

18. Totterdell P, Spelten E, Smith L, Baron J, Folkard S. Recovery from work shifts: how long does it take? J Appl Psychol 1995;80:43-57.

19. Frankenhaeuser M. Coping with job stress—a psychobiological approach. In: Gardell B, Johansson G, editors. Working life: a social science contribution to work reform. Chichester: John Wiley; 1981. p 213-33.

20. Rissler A. Stress reactions at work and after work during a period of quantitative overload. Ergonomics 1977;20:13-6.

21. House JS, Strecher V, Metzner HL, Robbins CA. Occupational stress and health among men and women in the Tecumseh community health study. J Health Soc Behav 1986;27:62-77.

22. Maruyama S, Morimoto K. Effects of long workhours on lifestyle, stress and quality of life among intermediate Japanese managers. Scand J Work Environ Health 1996;22:353-9.

23. Raggatt PTF. Work stress among long-distance coach drivers: a survey and correlational study. J Organ Behav 1991;12:56579.

24. Theorell T, Karasek RA. Current issues relating to psychosocial job strain and cardiovascular disease research. J Occup Health Psychol 1996;1:9-26.

25. Steenland K. Shift work, long hours and cardiovascular disease. Occup Med 2000;15:7-68.

26. Baldwin PJ, Dodd M, Wrate RW. Young doctors' health: I. How do working conditions affect attitudes, health and performance? Soc Sci Med 1997;45:35-40.

27. Borg V, Kristensen TS. Psychosocial work environment and mental health among travelling salespeople. Work Stress 1999;13:132-43.

28. Emdad R, Belkic K, Theorell T, Cizinsky S. What prevents professional drivers from following physicians' cardiologic advice? Psychother Psychosom 1998;67:226-40.

29. Ettner SL, Grzywacs JG. Workers' perceptions of how jobs affect health: a social ecological perspective. J Occup Health
Psychol 2001;6:101-13.

30. Hayashi T, Kobayashi Y, Yamaoka K, Yano E. Effect of overtime work on 24-hour ambulatory blood pressure. J Occup Environ Med 1996;38:1007-11.

31. Iwasaki K, Sasaki T, Oka T, Hisanaga N. Effect of working hours on biological functions related to cardiovascular system among salesmen in a machinery manufacturing company. Ind Health 1998;36:361-7.

32. Jex SM, Bliese PD. Efficacy beliefs as a moderator of the impact of work-related stressors: a multilevel study. J Appl Psychol 1999;84:349-61.

33. Kageyama T, Nishikido N, Kobayashi T, Kurokawa Y, Kaneko T, Kabuto M. Long commuting time, extensive overtime, and sympathodominant state assessed in terms of short-term heart rate variability among male white-collar workers in the Tokyo megalopolis. Ind Health 1998;36:209-17.

34. Kageyama T, Nishikido N, Kobayashi T, Kawagoe H. Estimated sleep debt and work stress in Japanese white-collar workers. Psychiatr Clin Neurosci 2001;55:217-9.

35. Kawakami N, Araki S, Takatsuka N, Shimizu H, Ishibashi H. Overtime, psychosocial working conditions, and occurrence of non-insulin dependent diabetes mellitus in Japanese men. J Epidemiol Community Health 1999;53:359-63.

36. Krause N, Lynch J, Kaplan GA, Cohen RD, Goldberg DE, Salonen JT. Predictors of disability retirement. Scand J Work Environ Health 1997;23:403-13.

37. Mure K, Takeshita T, Takeuchi T, Morimoto K. Urinary mutagens and lifestyle factors. Prev Med 1996;25(5):569-74.

38. Nakamura K, Shimai S, Kikichi S, Takahashi H, Tanaka M, Nakano $\mathrm{S}$, et al. Increases in body mass index and waist circumference as outcomes of working overtime. Occup Med 1998;48:169-73.

39. Nakanishi N, Nakamura K, Ichikawa S, Suzuki K, Tatara K. Lifestyle and the development of hypertension: a 3-year follow-up study of middle-aged Japanese male office workers. Occup Med 1999;49:109-14.

40. Nakanishi N, Yoshida H, Nagano K, Kawashimo H, Nakamura K, Tatara K. Long working hours and risk for hypertension in Japanese male white collar workers. J Epidemiol Community Health 2001;55:316-22.

41. Nylén L, Voss M, Floderus B. Mortality among women and men relative to unemployment, part time work, overtime work, and extra work: a study based on data form the Swedish twin registry. Occup Environ Med 2001;58:52-7.

42. Proctor SP, White RF, Robins TG, Echeverria D, Rocskay AZ. Effect of overtime work on cognitive function in automotive workers. Scand J Work Environ Health 1996;22:124-32.

43. Rosenstock SJ, Andersen LP, Rosenstock CV, Bonnevie O, Jorgensen T. Socioeconomic factors in heliobacter pylori infection among Danish adults. Am J Public Health 1996;86: 1539-44.

44. Sasaki T, Iwasaki K, Oka T, Hisanaga N, Ueda T, Takada Y, et al. Effect of working hours on cardiovascular-autonomic nervous functions in engineers in an electronics manufacturing company. Ind Health 1999;37:55-61.

45. Sasaki T, Iwasaki K, Oka T, Hisanaga N. Association of working hours with biological indices related to the cardiovascular system among engineers in a manufacturing company. Ind Health 1999;37:457-63.

46. Sokejima S, Kagamimori S. Working hours as a risk factor for acute myocardial infarction in Japan: case-control study. BMJ 1998;317:775-80.

47. Steptoe A, Wardle J, Lipsey Z, Mills R, Oliver G, Jarvis M, et al. A longitudinal study of workload and variations in 
psychological well-being, cortisol, smoking, and alcohol consumption. Ann Behav Med 1998;20(2):84-91.

48. Trinkoff AM, Storr CL. Work schedule characteristics and substance use in nurses. Am J Ind Med 1998;34:266-71.

49. Tyssen R, Vaglum P, Grønvold NT, Ekeberg Ø. The impact of job stress and working conditions on mental health problems among junior hours officers: a nationwide Norwegian prospective cohort study. Med Educ 2000;34:374-84.

50. Tyssen R, Vaglum P, Grønvold NT, Ekeberg Ø. Suicidal ideation among medical students and young physicians: a nationwide and prospective study of prevalence and predictors. J Affect Disorders 2001;64:69-79.

51. Voss M, Floderus B, Diderichsen F. Physical, psychosocial, and organisational factors relative to sickness absence: a study based on Sweden Post. Occup Environ Med 2001;58:178-84.

52. Yasuda A, Iwasaki K, Sasaki T, Oka T, Hisanaga N. Lower percentage of CD65+ cells associated with long working hours. Ind Health 2001;39:221-3.

53. Epstein RL. The effect of overtime work on blood pressure. J Occup Environ Med 1997;39:286.

54. Kageyama T, Nishikido N, Kobayashi T, Kurokawa Y, Kabuto M. Commuting, overtime, and cardiac autonomic activity in Tokyo. Lancet 1997;350:639.

55. Theorell T. Psychosocial cardiovascular risks - on the double loads in women. Psychother Psychosom 1991;55:81-9.

56. Nakano $Y$, Nakamura $S$, Hirata $M$, Harada $K$, Ando $K$, Tabuchi T, et al. Immune function and lifestyle of taxidrivers in Japan. Ind Health 1998;36:32-9.

57. Meijman TF, Thunissen MJ, De Vries-Griever AGH. The after-effects of a prolonged period of day-sleep on subjective sleep quality. Work Stress 1990;4:65-70.

58. Bangert-Drowns RL, Wells-Parker E, Chevillard I. Assessing the methodological quality of research in narrative reviews and meta-analyses. In: Bryant KJ, Windle M, West SG, editors. The science of prevention. Methodological advances from alcohol and substance abuse research. Washington (DC): American Psychological Association; 1997. p 405-29.

59. Beahler CC, Sundheim JJ, Trapp NI. Information retrieval in systematic reviews. Am J Prev Med 2000;18 Suppl 4:6-10.
60. Moncrieff J. Research synthesis: systematic reviews and metaanalysis. Int Rev Psychiatry 1998;10:304-11.

61. Wagenaar AC. Importance of systematic reviews and metaanalyses for research and practice. Am J Prev Med 1999;16 Suppl 1:9-11.

62. Fredriksson K, Alfredsson L, Köster M, Bildt Thorbjörnsson C, Toomingas A, Torgén M, et al. Risk factors for neck and upper limb disorders: results of 24 years of follow up. Occup Environ Med 1999;56:59-66.

63. Bildt Thorbjörnsson C, Alfredsson L, Fredriksson K, Michélsen H, Punnett L, Vingård E, et al. Physical and psychosocial factors related to low back pain during a 24-year period. Spine 2000;25:369-75.

64. Lundberg U, Palm K. Workload and catecholamine excretion in parents of preschool children. Work Stress 1989;3:255-60.

65. Sluiter JK, Frings-Dresen MHW, van der Beek AJ, Meijman TF, Heisterkamp SH. Neuroendocrine reactivity and recovery from work with different physical and mental demands. Scand J Work Environ Health 2000;26:306-16.

66. Nicolson NA, Van Diest R. Salivary cortisol patterns in vital exhaustion. J Psychosom Res 2000;49:335-42.

67. Sluiter JK, Frings-Dresen MHW, Van der Beek AJ, Meijman $\mathrm{TF}$. The relation between work-induced neuroendocrine reactivity and recovery, subjective need for recovery, and health status. J Psychosom Res 2001;50:29-37.

68. Pickering TG, Devereux RB, James GD, Gerin W, Landsbergis P, Schnall PL, et al. Environmental influences on blood pressure and the role of job strain. J Hypertens 1996;14 Suppl 5:179-85.

69. Haerenstam A, Theorell T. Cortisol elevation and serum $\gamma$-glutamyl transpeptidase in response to adverse job conditions: how are they interrelated? Biol Psychol 1990;31:15771.

70. Kawakami N, Araki S, Haratani T, Hemmi T. Relations of work stress to alcohol use and drinking problems in male and female employees of a computer factory in Japan. Environ Res 1993;62:314-24.

Received for publication: 11 July 2002 